\title{
A Rational Approach to Funding Your Research Enterprise
}

Douglas A. Girod, Executive Vice Chancellor, University of Kansas Medical Center; Executive Dean, University of Kansas School of Medicine

Paul Terranova, Vice Chancellor for Research, University of Kansas Medical Center Clay Tellers, Principal, Academic Healthcare Division, ECG Management Consultants

7 The rapidly changing financial environment of Academic Medical Centers (AMCs) has put increasing pressure on organizations to carefully evaluate the utilization of resources to maximize institutional priorities. Most public AMCs have multiple complex financial arrangements that provide the resources to meet the missions of education, research, service and clinical care. These sources include State funding (for public AMCs), Federal research funding, Industry research funding and contracts, student tuition and fees, mission support from affiliated hospitals, philanthropy, and clinical revenue from direct patient care. In recent years, possibly for the first time, all of these revenue streams have simultaneously come under increasing downward pressure.

The current state of institutional resource allocation to departments at many AMCs is largely historical in nature and developed over many years. Often these are based on Chair and faculty recruitment packages, prior institutional priorities, obsolete educational models and outdated faculty compensation plans. Many institutions are or have been working to put better definition to the allocation of resources in response to the ever increasing economic challenges facing our AMCs.

Not uncommonly, the education, research and service components of the enterprise are not self-sustaining and therefore need significant subsidization from other sources of revenue. While all missions are critical to the success of the
AMC, resources are inherently limited and therefore dictate the potential size and sustainability of all missions.

For KUMC, like most AMCs, the research effort reflects the diversity of funding sources seen in the other mission areas; usually the largest funding source remains the Federal Government in all its forms and the National Institutes of Health in particular. (Figure 1) Other Federal funding agencies include the National Cancer Institute, the Veterans Affairs Agency, the Center for Medicare and Medicaid Services, and The Department of Defense.

In the years since the international economic downturn of 2008, all these 


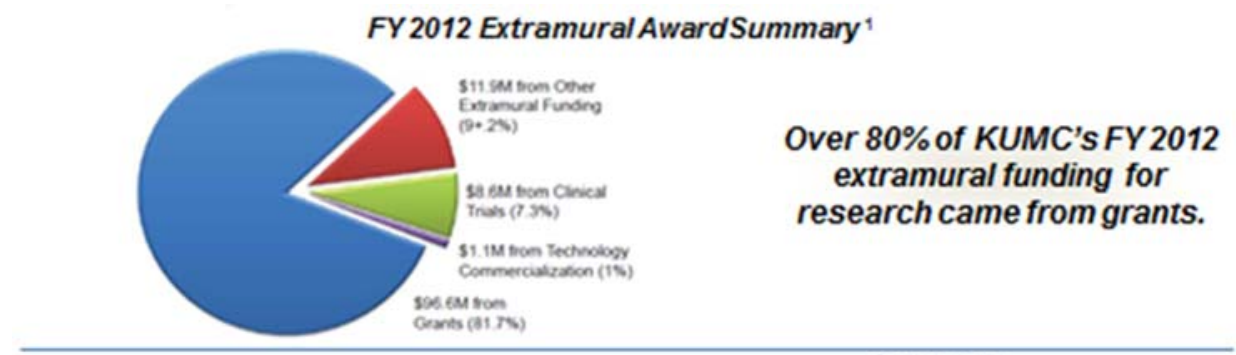

FY 2012 Awards by Funding Agency Type

Federal funding comprised nearly twothirds of KUMC's FY 2012 extramural awards.

'Source: KUMC Researoh insthate FY 2012 Amual Report.

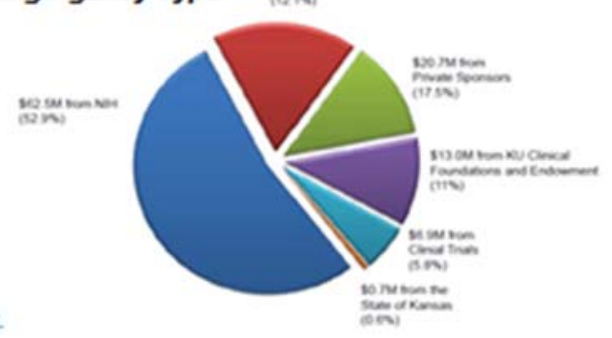

Figure 1: Overview of KUMC Extramural Research Funding Sources for FY2012

agencies have experienced major reductions or flattening of their budgets despite the inflation of expenses. This has resulted in fewer dollars to invest in research. For example, after the doubling of the NIH budget between 1995 and 2003 there has been a flattening and then reduction in the budget. Adjusting for inflation, the NIH funding level is at its lowest level since 2000. (Figure 2) As a consequence the percentage of NIH grant applications that are being funded is at the lowest level in history, and in the single digits for most of the Institutes. AMCs are witnessing even their most senior and experienced investigators losing much or all their extramural funding at a rate never before encountered.

Simultaneously, State budgets across the country have had to reduce significantly in response to diminished tax revenue as the economy has shrunk and the demand for services including unemployment have escalated. This has resulted in decreased funding for higher education. Student tuition and fees in our
AMCs are already at levels resulting in tremendous student debt burden and have little room for adjustment. Most AMCs also have many fewer students than the typical undergraduate University and therefore student tuition makes up a much smaller portion of the revenue stream.

Similarly, the economic downturn has resulted in a reduction of industry funded research and development and external contracts awarded. As individual and foundation investment funds suffered major losses in 2008 and still have not fully recovered, the amount of philanthropic dollars available to institutions has also been challenged.

As a consequence of these increasing challenges to funding of the multiple missions of the AMC, the University of Kansas Medical Center has undertaken a comprehensive review of all of their funding sources, and all expenditures 


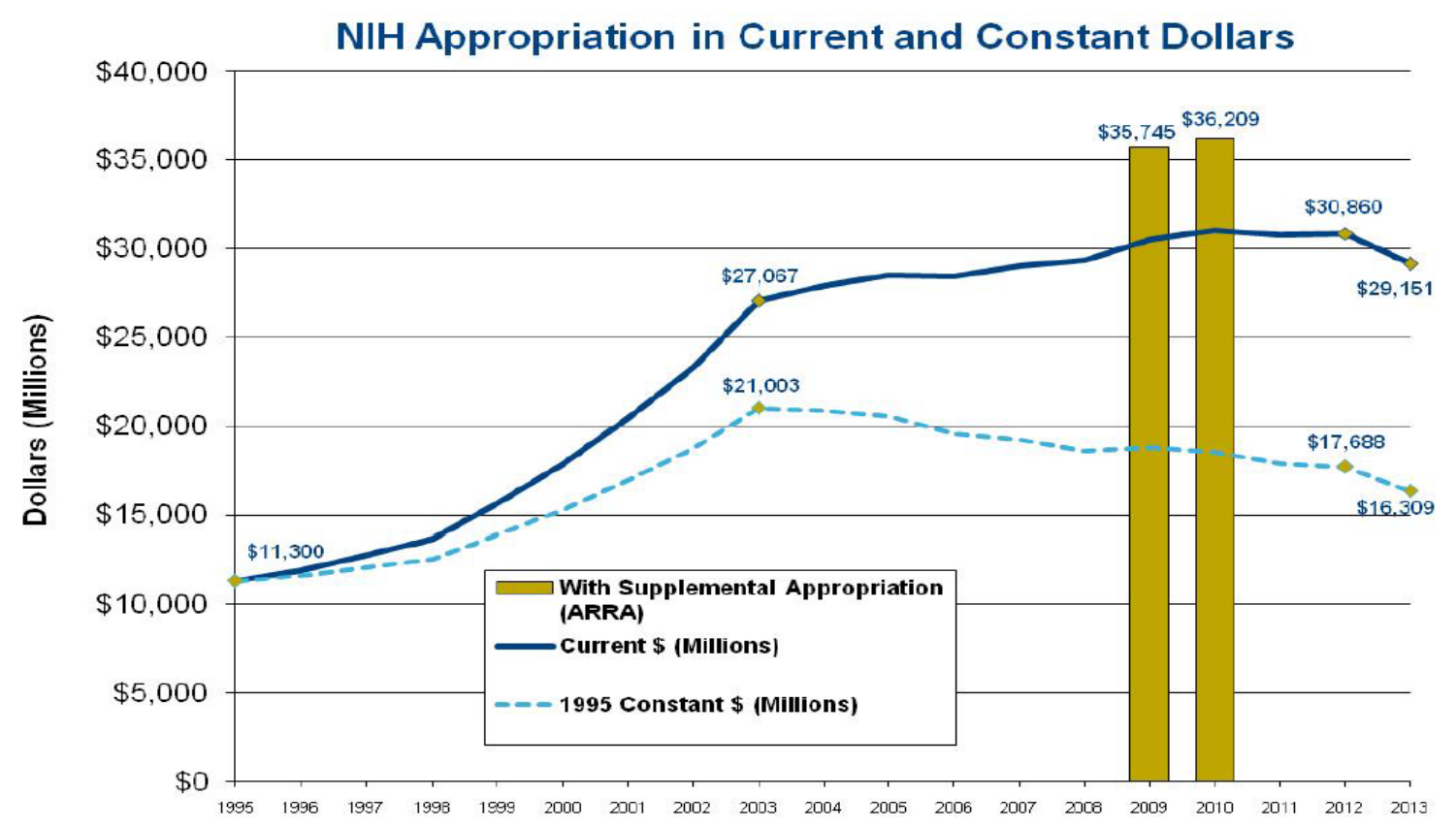

Figure 2

based on specific missions (education, research, service and clinical care). To fully understand whether our allocation of institutional resources reflects our mission priorities we needed to first understand the underlying cost of meeting each of our missions. This required the development of rational and reproducible funding models for each of the missions.

Therefore, each mission could not be examined in isolation but rather as part of a comprehensive design of the funding model. (Figure 3) This effort included building models for education funding (undergraduate, undergraduate medical, graduate and graduate medical education), faculty service funding, and research funding. In the case of KU medical center, the clinical enterprise underwent a separate process for the development of a funding model with the understanding that the clinical enterprise needed to fund the clinical mission.

The objectives of this effort specific to the research mission are outlined below:

- Provide a reasonable level of support for research while encouraging research programs to acquire extramural funding.

- Improve alignment between allocations of institutional funds and intended purpose/mission of those financial resources.

- Develop incentives for increasing faculty salary coverage from grants and other extramural sources.

- Recognize the fiscal realities and potential long-term impact of reduced/static federal funding and protect recent investments in research programs.

The entire effort to construct a comprehensive funding model encompassing all missions was to ultimately drive Institutional resource allocation as directed by the model and fully understand the expense associated with new programs, new hires and new research efforts. This 


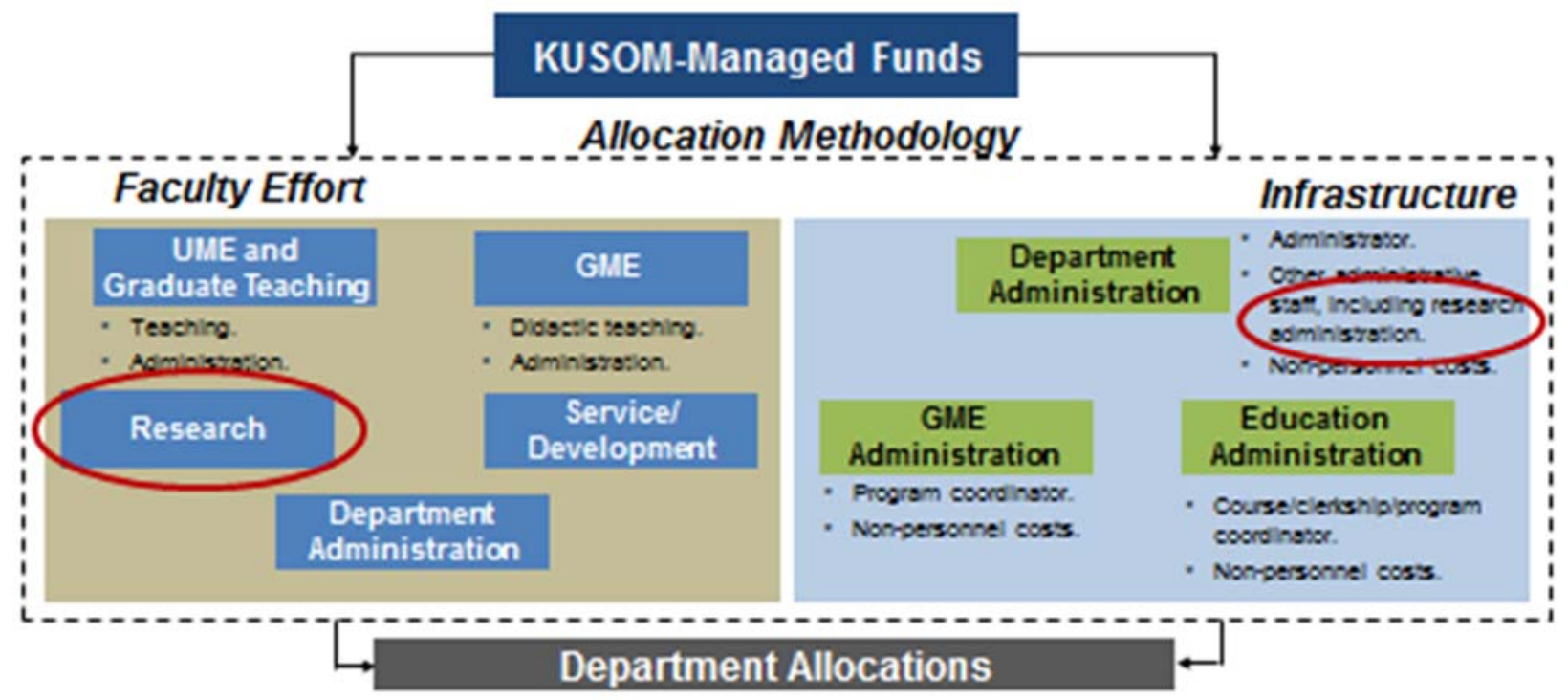

Figure 3: Comprehensive Funding Model approach for Institutional Resources for the KU School of Medicine. The research specific elements of the funding model are circled. (UME- Undergraduate Medical Education; GME=Graduate Medical Education; SOM=School of Medicine) model also would provide insight into what the current efforts in all mission areas were costing and inform decisions as to areas for elimination or expansion. This more expansive approach to our financial overview would provide the basis for directing resource allocation in line with our strategic plan and priorities.

\section{Methods}

Over the course of many months beginning September 2012 The University of Kansas Medical Center engaged ECG Management Consultants, Inc. to assist in the development of a comprehensive funding model that would incorporate education, research and service. The initial effort focused on the KU School of Medicine Kansas City campus with plans to extrapolate to the other component schools and campuses of KUMC. A stepwise approach with progressive institutional constituent engagement was undertaken as outlined below.

- Development of initial categories and assumptions based on ECG Management Consultants, Inc., experience at other institutions, with consideration of previous internal KUSOM development efforts.

- Large group meeting presentations to chairs, center/institute directors, and other key stakeholders, with subsequent feedback.

- Individual chair meetings to review data inputs and assumptions.

- Committee of non-clinical Chairs to address research compensation

- Ongoing weekly meetings with Office of Medical Education leadership to review and refine assumptions for the education model.

- Periodic evaluation sessions with EVC leadership team to review initial results, improve assumptions and allocation categories, and develop a potential implementation strategy.

As shown in Figure 3, this model contained a faculty effort component and a department administration component. 


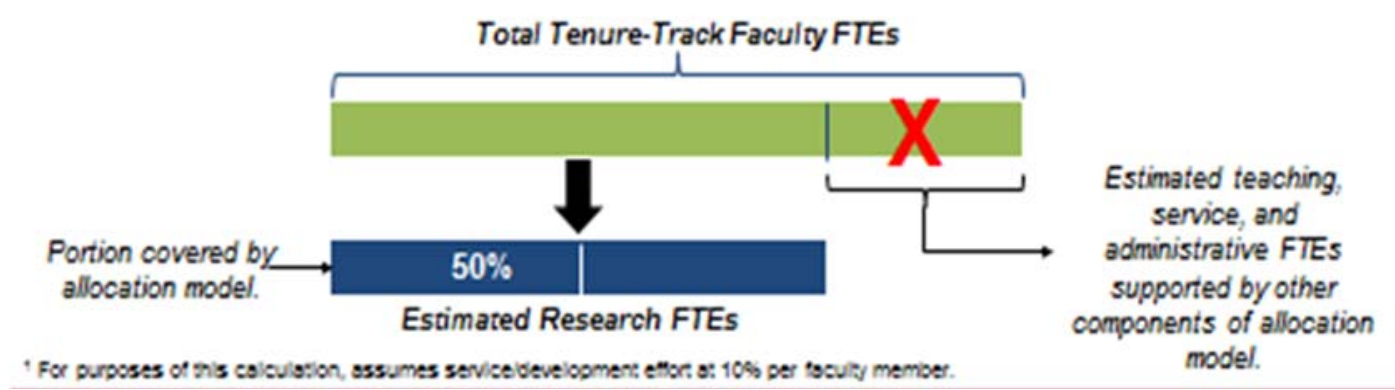

Figure 4: Basic Science and Academic Departments; methodology for determining the faculty research FTE at the department level.

The centralized expense components (Dean's office, finance, HR, facilities, etc.) were not part of the model development. Startup packages and grant bridging efforts were also excluded from the calculation and would represent funding above and beyond the model.

It was also determined by the oversight team (KUMC leadership and ECG) that the research component of clinical departments was inherently different than that of the basic science and other academic departments (biostatistics, health policy and management, history of medicine) and therefore required somewhat separate assumptions.

\section{Results}

The expense methodology developed provides support for a portion of estimated faculty research effort, with the remainder expected to be covered by grants and/or other departmentally sourced funding. The research model was built at the department level, not at the individual faculty level but there was much discussion surrounding how to measure the research effort of the faculty.

Research Salary Funding for Basic Science and Academic Departments

The following methodology was selected specifically for the basic science and other academic departments (Figure 4):

- Estimates department research FTEs using a "1 minus" approach.

$>$ Calculated as total tenure-track faculty FTEs less estimated FTEs for education, service/development, and administration.

$>$ Assumes that non-tenure-track faculty (e.g., research assistant professor) support research effort from grants and/or other departmentally sourced funding.

- Allocates funding to support $50 \%$ of estimated faculty research FTEs, based on the AAMC Midwest median benchmark for an associate professor annual salary in the given specialty and a benefits rate of $25 \%$.

For the "1 minus" approach to work, the other components of the model needed to be developed first (education and service/development). For this model the service/development component was set at $10 \%$, the education component was calculated using a separate education funding model developed in a similar fashion. Thus any faculty time not committed to education or research as de- 


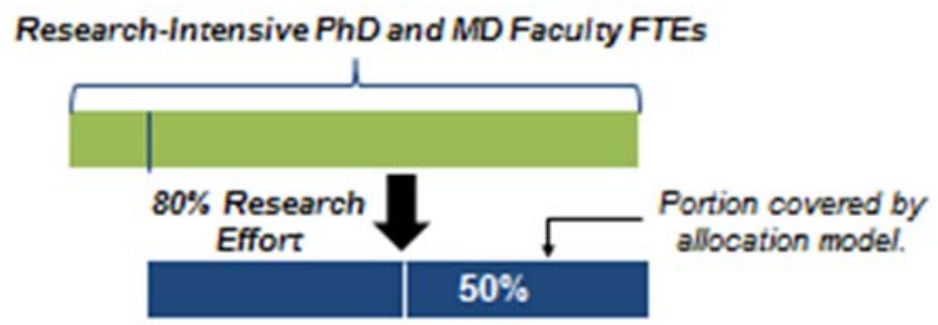

Figure 5: Clinical Departments; methodology for determining the faculty research FTE at the department level.

fined by the model is assumed to be Research effort. The sum of all the research effort for a given department is what ultimately determined the research expense (and will subsequently drive institutional resource allocation).

Research Salary Funding for Clinical Departments

As the research faculty in clinical departments are less engaged in education and more focused on research efforts it was decided to fix the research time commitment at $80 \%$ time for all research intensive faculty in the clinical departments as outlined below. (Figure 5) The model:

- Provides support for individuals identified as research-intensive (i.e., tenure-track, clinical scholar-track, and clinical educator-track faculty) who meet one of the following criteria:

$>$ Primary degree is $\mathrm{PhD}$.

Dalary coverage of $35 \%$ or more from grants.

- Assumes $80 \%$ research effort for faculty identified as research-intensive.

- Provides internal salary support for $50 \%$ of estimated research effort for all identified research-intensive faculty.

$>$ PhD Faculty - Valued at AAMC Midwest median benchmark for an associate professor in the basic sciences overall and a benefits rate of $25 \%$.

$>$ MD Faculty - Valued at AAMC Midwest median benchmark for an associate professor in the given specialty and a benefits rate of $25 \%$.

Allocations for Service/Development and Department Administration

Allocations for faculty effort in service/development efforts and overall department administration are determined based on the following assumptions 1) for departmental/school/medical center service and/or faculty development $\$ 12,500$ salary per faculty FTE is provided for tenure track faculty only (although consideration should be given to research track faculty who serve on committees with the School of Medicine/Medical Center); 2) the Chair would receive 0.1 administrative FTE for assistance with duties regardless of the department size and an addition 0.01 FTE per faculty member; the department would receive 3) 1 executive administrator FTE per 25 faculty FTEs, with a minimum of one FTE regardless of the department size and 4) one administrative assistant FTE per 10 faculty FTEs, with a minimum of one administrative assistant FTE per department; and 5) for non-personnel infra- 
structure (departmental OOE) $\$ 750$ is allocated per faculty FTE. The administrative support is responsible for supporting all the missions of the department including education, research and service. Additional research administration support is available centrally within the Research Institute.

\section{Model Simulation}

Once the model had been developed, the data for faculty effort in each department was verified with the departmental Chair and administrator. The research model was then simulated utilizing the criteria as outlined above and the data generated for all departments as a high level evaluation. The simulation suggests a funding need of $\$ 8$ million dollars for all departments in the School of Medicine for the support of the research mission. This was compared to a total of $\$ 17$ million of faculty salary currently placed on grants. Thus, it would appear that the model would in fact suggest matching roughly half of the salary time placed on grants. This is consistent with the assumption that the Institution would support $50 \%$ of the research time effort.

\section{Discussion}

This paper outlines the efforts of the University of Kansas School of Medicine to develop a rational and reproducible funding model for the allocation of Institutional resources for the defined purpose of supporting the research mission. This effort was undertaken as an element of a more comprehensive funding model project that also including funding allocations for the education and service mission areas.

A transparent and collaborative process was utilized to engage institutional and departmental leaders in the development of the model. Through the course of the process this input was critical in identifying elements of the model or unique situations in the institution that needed to be incorporated or modified to be truly representative of the research efforts. This process has also facilitated the "buy in" of the leaders in the model.

A first pass high level simulation of the model would suggest a level of funding at about $47 \%$ of the amount of salary currently placed on grants for research faculty effort. In other words, this does seem to model roughly $50 \%$ of the faculty research effort as envisioned by the model. Thus it would appear to achieve the targeted goal.

The funding allocation model is developed at the departmental level. It is envisioned that it will continue to be the responsibility of the department Chairs to manage the actual allocation of the funds in the context of the other sources of funding available (research grants and contracts, service agreements, educational and service mission funding, indirect returns from the institutional portion of grant funding, and clinical revenue for the Clinical departments). Since the model is based on Associate Professor AAMC salary benchmarks, the actual distribution of the faculty in a given department may differ.

The model does offer an incentive for research success within a department. To the degree that more than $50 \%$ of a given department's faculty research effort is supported by other sources (grants, etc.), the actual institutional funding allocation in the model remains the same. Therefore, additional funds are available 
within the department for investment opportunities.

There are multiple advantages of developing a straightforward rational approach to defining the funding allocation model for our institution:

- Clearly defined and predictable approach for research funding for departments.

- Defines the "rules" for success.

- Facilitates the evaluation of new research "investments".

- Puts accountability on the Chairs to best manage resources.

Also contained in this model is an incentive to aggressively manage faculty that are struggling to adequately fund their research effort. If the collective department research effort grant support falls below $50 \%$ there are not increased allocated funds to fill the gap. This is much more manageable given that the other missions (education and service/development) have independent allocation methods for support.

As with any allocation methodology there are also some potential disadvantages:

- The model relies on faculty numbers and research effort and therefore changes with every new faculty member

- The model will need to be revisited on a regular basis to account for new faculty coming off startup packages etc.

- The model does not take into account the need for bridging of faculty salary and research expenses in the setting of lost grant funding. A bridging policy will be a necessary complement to the allocation model.
- Institutional resources are not always predicable in the current dynamic economic environment and therefore may not be adequate in the future to fully fund the model.

The successful implementation of the model will require a complete understanding of the key elements by Chairs and faculty alike. A result of developing the model at the departmental level allows for the Chair to manage the department budget to account for the idiosyncrasies of a given department yet sets clear accountability to the Institution for meeting all the required missions with the given funding allocations.

Once the model is run at the departmental level there will likely be variations between the funding allocation dictated by the model and the current funding allocations which are largely historical in nature. It is anticipated that if variations of more than $10 \%$ occur a staged adjustment over a few years will be necessary to avoid major programmatic disruptions. These adjustments will need to occur in the course of the normal institutional budget cycle.

\section{Conclusion}

An institutional funding allocation model to support the research mission at our AMC has been developed that is based on rational, reasonable and well defined elements agreed upon by the institutional leaders and department Chairs. First pass high level simulation of the model would suggest that the model is successful at defining support for $50 \%$ of the faculty research effort as intended. The model contains incentives for successful extramural funding yet holds departments and their leaders accountable to manage resources to meet all the missions of the institution. 